\title{
New research in kidney cancer, ASCO 2017
}

\author{
Naveen S. Basappa, MD, FRCPC'; Anil Kapoor, MD, FRCSC 2 \\ 'Cross Cancer Institute \& University of Alberta, Edmonton, AB; ${ }^{2}$ CUAJ Associate Editor \& McMaster University, Hamilton, ON; Canada
}

Cite as: Can Urol Assoc J 2017;11 (8Supp|5):S298-300.

http://dx.doi.org/10.5489/cuaj.4840

\section{First-line tyrosine kinase inhibitor therapy in metastatic disease}

Targeted therapy (e.g., with tyrosine kinase inhibitors [TKIs]) is the current preferred first-line treatment for advanced renal cell carcinoma (aRCC). ${ }^{1}$ One of the dominant themes in the use of TKIs in this setting continues to be optimization of dose and schedule to obtain the maximum benefit for each individual patient.

The head-to-head COMPARZ study comparing sunitinib and pazopanib was a randomized, phase 3 trial with primary efficacy and safety results published in the New England Journal of Medicine in 2013. ${ }^{2}$ The protocol for this study allowed for dose modifications and treatment interruptions based on tolerability. At ASCO 2017, investigators presented an analysis of the COMPARZ study data, stratified by the number of dose reductions or dose interruptions. ${ }^{3}$ The investigators reported that individuals who underwent dose reduction and/or interruption had higher mean cumulative doses of pazopanib or sunitinib, as well as longer time on treatment. For example, for those treated with pazopanib who did not undergo any dose reduction, the median cumulative dose and time on therapy were $134.4 \mathrm{~g}$ and 5.6 months, while for those with two or more dose reductions, the medians were $150.2 \mathrm{~g}$ and 11.4 months, respectively.

The progression-free survival (PFS) also differed substantially based on the number of dose reductions (Fig. 1A) and treatment interruptions (Fig. 1B). Higher response rates were also observed for those individuals who underwent two or more dose modifications compared to those who did not have any modifications. For pazopanib, the response rate was $56 \%$ for those with $\geq 2$ dose reductions compared to $22 \%$ for those with none, and $54 \%$ for those with $\geq 2$ dose interruptions compared to $23 \%$ for those with none. For sunitinib, a similar pattern was observed. For those with $\geq 2$ dose reductions or interruptions, the response rates were $37 \%$ and $39 \%$, respectively, while for those with none, the rates were $16 \%$ and $16 \%$, respectively.

The COMPARZ study database was also analyzed in an effort to characterize those patients who achieved a long-term response (LTR) on pazopanib or sunitinib. ${ }^{4}$ The investigators identified the subsets of patients who had an LTR of 10 months or longer and 18 months or longer and compared their baseline characteristics, including age, Karnofsky performance status (KPS), number of metastatic sites, number of organs involved and Memorial Sloan Kettering Cancer Centre (MSKCC) risk category. The proportions of patients who achieved an LTR were similar for both pazopanib and sunitinib. The investigators did note, however, that the median time to response (complete response [CR] or partial response [PR]) was numerically shorter with pazopanib (11.9 weeks, 95\% confidence interval [CI] 11.3-12.1) compared to sunitinib (17.4 weeks, 95\% Cl 12.7-18.0; Table 1); however, using logistic regression analysis, the investigators reported that none of the examined baseline characteristics were significant predictors of response to either pazopanib or sunitinib.

Sunitinib dose modification was prospectively analyzed in two phase 2 studies presented at ASCO 2017.5,6 In one study, the investigators hypothesized that by individualizing sunitinib dose based on toxicity, PFS could be substantially improved from the 8.5 months observed in the non-individualized EFFECT study. ${ }^{7}$ There were scheduled toxicity evaluations after two and four weeks of the first treatment cycle (starting dose of $50 \mathrm{mg}$ per day for 28 days) and investigators were instructed to continue to maximize the days on therapy based on toxicity and to minimize time off treatment to seven days - contrary to the standard 28 days on and 14 days off treatment, as per the sunitinib product monograph.

A total of 117 patients were enrolled in the study. Nine patients discontinued early (for toxicity [ $n=5]$, non-compliance $[n=2]$, and global deterioration $[n=2]$ ), leaving 108 patients evaluable for response. The proportion of patients who achieved an objective response (CR + PR) was $49.1 \%$ (Fig. 2). This was markedly higher than the $32 \%$ observed 


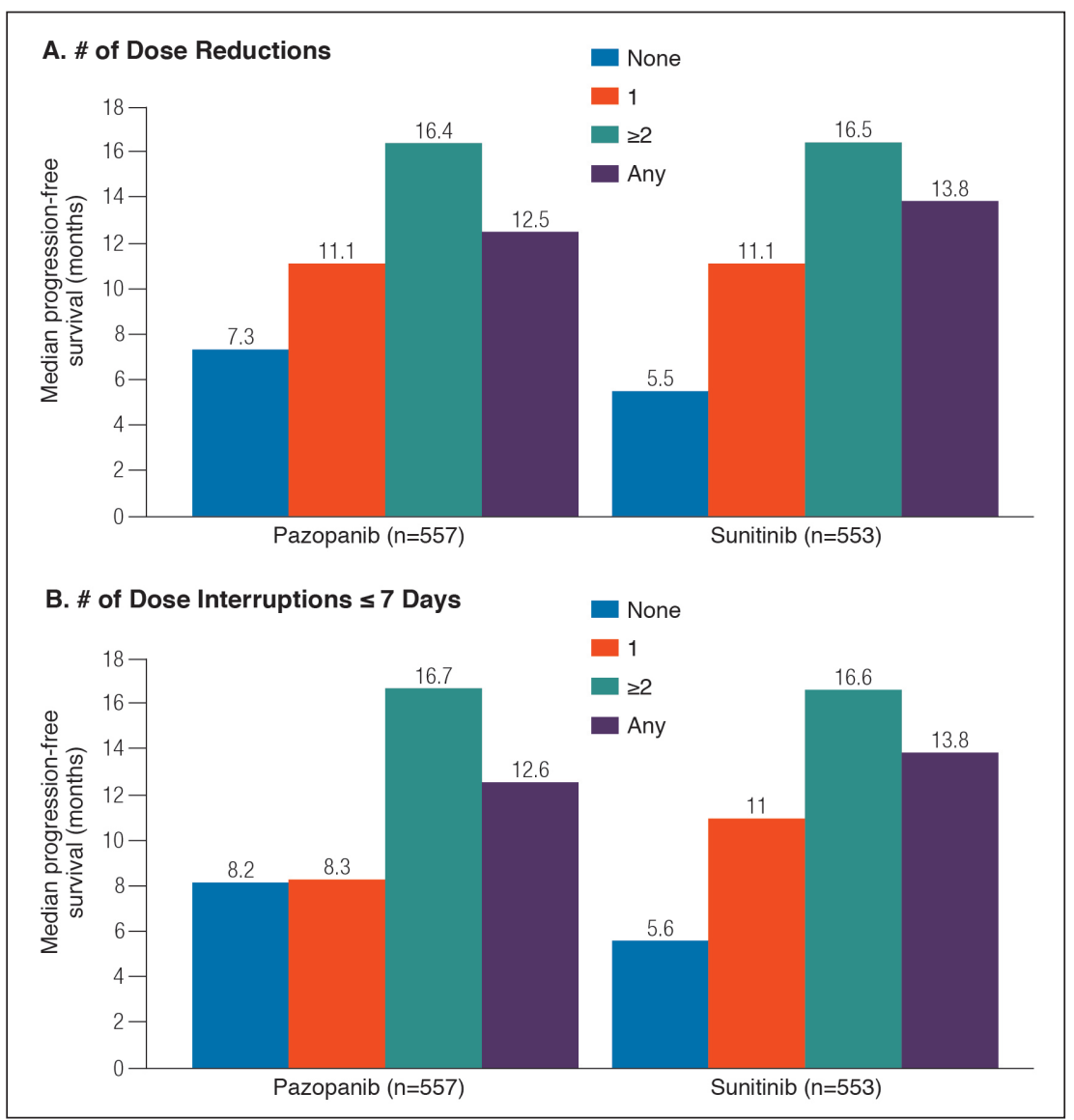

Fig. 1. Analysis of the COMPARZ study: Progression-free survival by number of $(\boldsymbol{A})$ dose reductions; or (B) treatment interruptions.

in the EFFECT study. The proportion with clinical benefit $(\mathrm{CR}+\mathrm{PR}+$ stable disease $[S D])$ was $91.7 \%$; the clinical benefit rate in EFFECT was $75 \%$.

PFS and overall survival (OS) were also markedly better in this individualized dosing study (12.5 months and 38.5 months, respectively) relative to the historical rates achieved in EFFECT (8.5 months and 23.1 months, respectively).

The second phase 2 study evaluating sunitinib dose optimization evaluated an alternative two-weeks-on/one-weekoff regimen (with dose reductions allowed per protocol) in 60 patients with previously untreated aRCC. The hypothesis was that this regimen would reduce the incidence of Grade 3 or higher selected adverse events (AEs: fatigue, diarrhea, or

\section{Table 1. Time to response (CR/PR) with pazopanib or} sunitinib: Subanalysis of the COMPARZ study

\begin{tabular}{|c|c|c|c|}
\hline & & Pazopanib & Sunitinib \\
\hline \multicolumn{2}{|c|}{$\begin{array}{l}\text { Number of patients achieving } \\
\text { CR/PR }\end{array}$} & 171 & 137 \\
\hline \multirow{3}{*}{$\begin{array}{l}\text { Time to } \\
\text { response, } \\
\text { weeks }(95 \% \mathrm{Cl})\end{array}$} & First quartile & $6.0(6.0-6.3)$ & $11.6(8.3-12.0)$ \\
\hline & Median & $11.9(11.3-12.1)$ & $17.4(12.7-18.0)$ \\
\hline & Third quartile & $17.6(12.9-18.1)$ & $30.1(23.1-35.9)$ \\
\hline
\end{tabular}

hand-foot syndrome) from the published standard rate of $25-30 \%$ with the four-week-on/ two-week-off regimen. ${ }^{6}$ While the investigators reported good efficacy with this regimen (55\% overall response; $95 \%$ clinical benefit, median PFS 13.7 months, and median OS not reached), which led them to conclude this regimen is a feasible strategy, there was no notable difference compared to previously published standard rates of the selected AEs.

Based on the above abstracts, it is clear that optimal dosing of patients is key in maximizing outcomes, but that to accomplish this, the dosing needs to be individualized on a patient-by-patient basis. Toxicity appears to be a biomarker for adequate dosing, irrespective of which TKI is being used, indicating a class effect vs. a drug-specific effect. As the data to support this practice have continued to grow - and now with prospective data available - an individualized approach to aRCC patients with dose and schedule alterations to maximize drug exposure can be considered the optimal way to maximize patient response.

\section{Adjuvant TKI therapy}

While the efficacy of targeted therapy in aRCC is well-established, the role of these agents for adjuvant use is less well-characterized. At ASCO 2017, researchers presented the results of a randomized, phase 3 study evaluating adjuvant pazopanib compared to placebo in patients with locally advanced RCC after nephrectomy at high risk for recurrence. ${ }^{8}$ This study (the PROTECT trial) enrolled a total of 1538 patients with resected pT2 (high-grade), pT3 or greater clear-cell RCC. Subjects were randomized to receive pazopanib or placebo for one year. The initial dose was $800 \mathrm{mg}$ daily, and 403 patients were enrolled using this protocol. The protocol was subsequently amended to investigate a starting dose of 600 mg daily dose (which could be increased to $800 \mathrm{mg}$ daily based on tolerability), and 1135 subjects were randomized following this amendment. The primary efficacy analysis was disease-free survival (DFS) in the ITT (600 mg) population $(n=571)$ vs. placebo $(n=564)$. For the primary analysis, there was no significant difference between the groups, with a hazard ratio (HR) of $0.86(95 \% \mathrm{Cl} 0.70-1.06)$. On-therapy Grade 3/4 AEs occurred in $60 \%$ of the active therapy arm and $21 \%$ of the placebo arm. Of note, the $800 \mathrm{mg}$ daily dose analysis did show a statistically significant DFS advantage (HR 0.69, 95\% Cl 0.51-0.94), but as this was a secondary analysis in the study; there was not sufficient power to make any conclusions on these data at this time. Based on 
the $600 \mathrm{mg}$ primary analysis, the investigators concluded that pazopanib was not recommended for adjuvant therapy in patients with resected locally advanced RCC at high risk for recurrence.

\section{References}

1. North SA, Basappa N, Basiuk J, et al. Management of advanced kidney cancer: Canadian Kidney Cancer Forum consensus update. Can Urol Assoc J 2015; 9:164-70. https://doi.org/10.5489/cuai.2894

2. Motzer RJ, Hutson TE, Cella D, et al. Pazopanib vs. sunitinib in metastatic renalcell carcinoma. N Engl J Med 2013;369:722-31. ht1ps://doi.org/10.1056/ NEJMoa1303989

3. Bjarnason GA, Kollmannsberger CK, Ahmad Q1, et al. Effects of pazopanib (PAZ) and sunitinib (SUN) dose modification on safety and efficacy in metastatic renal cell carcinoma (mRCC) patients from COMPARZ. Poster presented at ASCO 2017; abstract \#4574.

4. Tannir NM, Porta C, Grünwald V, et al. Long-term response and time to response to pazopanib (PAZ) and sunitinib (SUN) in metastatic renal cell carcinoma (mRCC): COMPARZ subanalysis. Poster presented at ASCO 2017; abstract \#4572.

5. Bjarnason GA, Knox JJ, Kollmannsberger CK, et al. Phase 2 study of individualized sunitinib (SUN) as first-line therapy for metastatic renal cell cancer. Poster presented at ASCO 2017; abstract \#4514.

6. Jonasch E, Slack R, Geynisman DM, et al. Phase 2 study of alternate sunitinib schedule in patients with metastatic renal cell carcinoma. Poster presented at ASCO 2017; abstract \#v4513. disease.

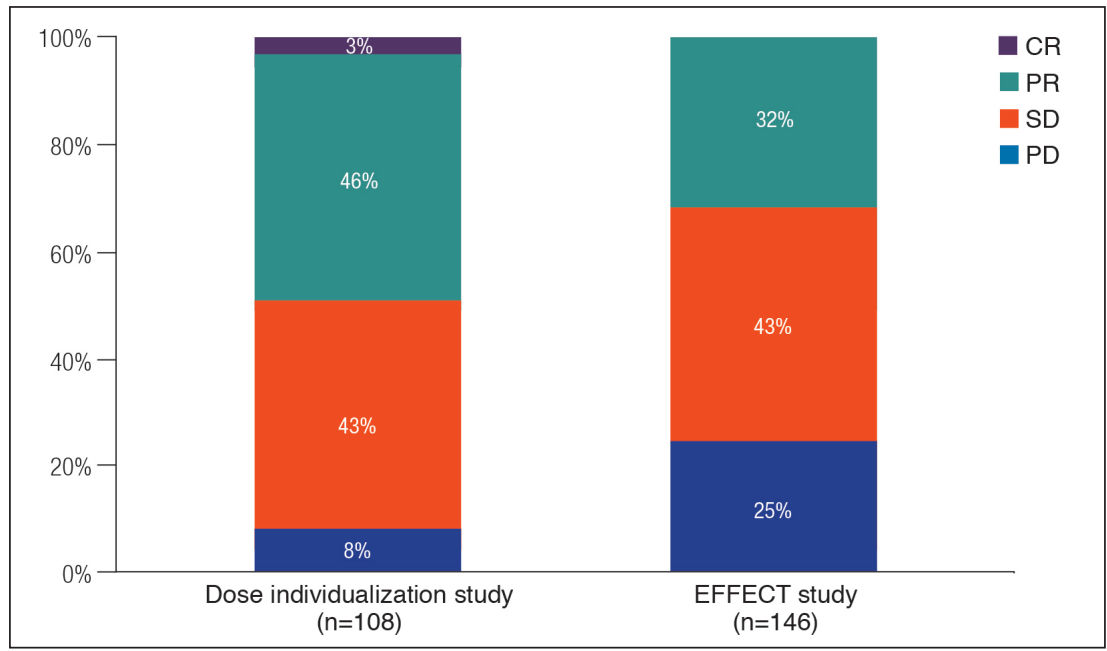

Fig. 2. Response to sunitinib in prospective dose individualization study (and historical comparison to EFFECT study). CR: complete response; PD: progressive disease; PR: partial response; SD: stable

7. Motzer RJ, Hutson TE, Olsen MR, et al. Randomized phase 2 trial of sunitinib on an intermittent vs. continuous dosing schedule as first-line therapy for advanced renal cell carcinoma. J Clin Oncol 2012; 30:1371-7. https://doi.org/10.1200/JC0.2011.36.4133

8. Motzer R, Haas N , Donskov F, et al. Randomized phase 3 trial of adjuvant pazopanib vs. placebo after nephrectomy in patients with locally advanced renal cell carcinoma (RCC) (PROTECT). Oral presentation presented at ASCO 2017; abstract \#4507. 